like packet with the middle layer made of softer (rubber-like) material it is proved appropriate to apply seismoisolations in seismosteady construction.

The asymptotic method may become the base for studying the interaction of plates and shells with different physical fields. The method is applicable for the solution of connected dynamic problems of thermoelasticity for isotropic and anisotropic plates.

Space dynamic problem of electroelasticity for a beforehand polarized piezoceramic plate is solved. Problems of straight and backward piezoelectric effects were considered. The asymptotic orders of the stresses tensor, displacements vector components, the electric field potential, the components of the vectors, correspondingly, the electric field strength and electric induction of piezoceramic plate are established. By an asymptotic method simple recurrent formulae, permitting to determine the displacement vector components, the stresses tensor and the stressness of the electric field with a beforehand given asymptotic exactness are derived. In general case different variants of space boundary conditions on the facial surfaces of the plate are satisfied. Dispersion equations for determination of frequencies values of outer effects, conducting to rise of resonances under shear and longitudinal vibrations are derived. The roots of these equations are found.

The analogous class of problems for piezoceramic shell, polarized along the thickness, is solved.

References
1. Aghalovyan L. A. Asymptotic Theory of Anisotropic Plates and Shells. Moscow. Nauka. $-1997 .-414$ p.

2. Aghalovyan L. A., Gevorgyan R. S. Nonclassical Boundary-value Problems of Anisotropic Layered Beams, Plates and Shells. Publishing House of the National Academy of Sciences of Armenia. Yerevan. - 2005.

3. Aghalovyan L. A. On one method of solution of three-dimensional dynamic problems for layered elastic plates and applications in seismology and seismosteady construction. Proceed. European Conference on Structural Control, 4ECSC. St. Peterburg. Russia. 2008. - Vol. 1. - P. 25 - 33.

\title{
ON THE THEORIES OF PLATES AND SHELLS AT THE NANOSCALE
}

\author{
Altenbach H. ${ }^{*}$, Eremeyev V. A. ${ }^{*} \dagger$ \\ * Otto-von-Guericke University Magdeburg \\ Universitatsplatz 2, 39106 Magdeburg, Germany \\ holm.altenbach@ovgu.de \\ †South Scientific Center of RASci\& South Federal University \\ Rostov on Don, Russia \\ eremeyev.victor@gmail.com
}

During the last 50 years the nanotechnology is established as one of the advanced technologies manipulating matter on an atomic and molecular scale. By this approach new materials, devices or other structures possessing at least one dimension sized from 1 to $100 \mathrm{~nm}$ are developed. As usual if the size decreases some ef- 
fects (for example, surface stresses) are more significant in comparison with effects of materials and devices of conventional size. Such effects are statistical mechanical and quantum mechanical effects among others. They results in significant changes of the properties in comparison with the properties of the bulk material (see [1-4] among others). This is experimentally established also for the inelastic properties, see, for example, $[5,6]$.

With respect to several applications resulting, for example, in new material properties the questions arise:

- how to model such nanostructures,

- how to design nanodevices, and

- how to predict the new properties.

Two approaches are suggested

-theories which take into account quantum mechanical effects since they are important at the quantum

-theories which are based on the classical continuum mechanics adapted to nanoscale problems.

In recent publications various approaches of modeling are presented. Many contributions are coming from physics (first approach) and yields in equations which are not similar to the usual equations in the engineering analysis of structures or engineering simulations of the material behavior. By this way we get results describing qualitatively and quantitatively correct the effects related to the nanosize. Since nanoeffects are connected with the size of atoms and molecules molecular modeling codes are used in numerical simulations. The disadvantage of this approach is that the engineering analysis of real nanostructures taking into account the three-dimensional behavior is not trivial. So in engineering applications the second approach is preferred. In contrast to the aforementioned approach in various applications Continuum Mechanics based theories are applied. It can be shown that the classical Continuum Mechanics is unable to simulate the properties of nanostructures in a correct manner [7-9]. Several improved theories for the analysis of nanostructures are developed, for example,

- Cosserat or micropolar theories,

- Continuum Mechanics theories taking into account surface effects, and

- Gradient theories.

It should be noted that these theories can be combined with the classical structural analysis introducing models like beam, rod, plate, shell, etc. as basic one- or two-dimensional models. Now there are developed several theories like, for example, the mechanics of nanocomposites which is widely discussed in the literature (cp. the review [10]). Alternatively, the behavior of the crystal lattice is modeled based on Continuum Mechanics, for example, in [11, 12].

Below we present the basic features of the classical Continuum Mechanics and discuss one possible improvement (the account of surface effects) which is helpful in nanomechanics. Gradient and Cosserat theories will be not discussed. They are presented in [13-16] among others. 


\section{References}

1. Chen C. Q., Shi Y., Zhang Y. S., Zhu J., Yan Y. J. Size dependence of Young's modulus in ZnO nanowires // Phys. Rev. Lett. - 2006. -Vol. 96, 075, 505. - P. 1 - 4.

2. Cuenot S., Fru $\square$ tigny C., Demoustier-Champagne S., Nysten B. Surface tension effect on the me-chanical properties of nanomaterials measured by atomic force microscopy // Phys. Rev. B 69. - 2004. - Vol. 165, 410. P. 1-5.

3. Greer J. R., Nix W. D. Size dependence of mechanical properties of gold at the submicron scale // Appl. Phys. - 2005. - Vol. A 80. - P. 1625 - 1629.

4. Jing G. Y., Duan H. L., Sun X. M., Zhang Z. S., Xu J., Li Y. D., Wang J. X., Yu D. P. Surface effects on elastic properties of silver nanowires: Contact atomic-force microscopy // Phys. Rev. B 73(23). - 2006. Vol. 235. P. 409 - 235, 416.

5. Fleck N. A., Muller G. M., Ashby M. F., Hutchinson J. W. Strain gradient plasticity: Theory and experiment // Acta Metall. Mater. - 1992. - Vol. 42 (2). - P. 475 - 487.

6. Stolken J. S., Evans A. G. Microbend test method for measuring the plasticity length scale // Acta Mater. - 1998. - Vol. 46 (14). - P. 5109 - 5115.

7. Govindjee S., Sackman J. L. On the use of continuum mechanics to estimate the properties of nan- otubes // Solid State Commun. - 1999. - Vol. 110 (4). - P. 227 - 230.

8. Gurtin M. E., Markenscoff X., Thurston R. N. Effect of surface stress on the natural frequency of thin crystals // Appl. Phys. Lett. - 1976. - Vol. 29 (9). - P. 529 - 530.

9. Zhou L. G., Huang H. Are surfaces elastically softer or stiffer // Appl. Phys. Lett. 2004. - Vol. 84 (11). - P. 1940 - 1942.

10. Guz A. N., Rushchitsky J. J. Establishing foundations of the mechanics of nanocomposites (review) // Int. Appl. Mech. - 2011. - Vol. 47 (1). - P. 2 - 44.

11. Alizada A. N., Sofiyev A. H. Modified Young's moduli of nano-materials taking into account the scale effects and vacancies // Meccanica. - 2011. - Vol. 46. - P. 915 920.

12. Krivtsov A. M., Morozov N. F. On mechanical characteristics of nanocrystals // Phys. Solid State. - 2002. - Vol. 44 (12). - P. 2260 - 2065.

13. Aifantis E. Update on a class of gradient theories // Mech. Mater. - 2003. - Vol. 35 (3-6) - P. $259-280$.

14. Asghari M. Geometrically nonlinear micro-plate formulation based on the modified couple stress theory // Int. J. Eng. Sci. - 2012. - Vol. 51 (18). - P. 292 - 309.

15. Chen S. H., Feng B. Size effect in micro-scale cantilever beam bending // Acta Mech. 2011. - Vol. 219. - P. $291-307$.

16. Lazopoulos K. A. On the gradient strain elasticity theory of plates // Europ. J. Mech. A/Solids. - 2004. - Vol. 23 (5). - P. 843 - 852.

\section{A SHELL THEORY FOR CNTs OF ARBITRARY CHIRALITY \\ Podio-Guidugli P. \\ DICII, University of Rome TorVergata, Via Politecnico, 1 - 00133 Roma (Italy) ppg@uniroma2.it}

Summary. Chirality is a geometrical character that influences heavily the mechanical, electrical, and thermal, properties of a CNT, especially when its radius is small; in particular, a variety of chirality-dependent mechanical phenomenologies is described in $[6,2,5,9,13,14]$. In this talk, which is based on joint work with 